\title{
DERIVING EXCLUSION MAPS FROM C-BAND SAR TIME-SERIES: AN ADDITIONAL INFORMATION LAYER FOR SAR-BASED FLOOD EXTENT MAPPING
}

\author{
Jie Zhao ${ }^{1,2,}{ }^{*}$, Marco Chini ${ }^{1}$,Ramona Pelich ${ }^{1}$, Patrick Matgen ${ }^{1}$, Renaud Hostache ${ }^{1}$, Senmao Cao ${ }^{2}$, Wolfgang Wagner ${ }^{2}$ \\ ${ }^{1}$ Department of Environmental Research and Innovation, Luxembourg Institute of Science and Technology, Luxembourg - (jie.zhao, \\ marco.chini, ramona.pelich, Patrick.matgen, Renaud.hostache)@list.lu \\ ${ }^{2}$ Research Group Remote Sensing, Department of Geodesy and Geoinformation, Vienna University of Technology, Austria - \\ (senmao.cao, wolfgang.wagner) @geo.tuwien.ac.at
}

KEY WORDS: Sentinel-1, exclusion map, flood mapping, shadow, layover, urban, densely vegetated area

\begin{abstract}
:
Change detection has been widely used in many flood-mapping algorithms using pairs of Synthetic Aperture Radar (SAR) intensity images as floodwater often leads to a substantial decrease of backscatter. However, limitations still exist in many areas, such as shadow, layover, urban areas and densely vegetated areas, where the SAR backscatter is not sufficiently impacted by floodwater-related surface changes. This study focuses on these so-called exclusion areas, i.e. areas where SAR does not allow detecting water based on change detection. Our approach considers both pixel-based time series analyses and object-based spatial analyses using $20 \mathrm{~m}$ Sentinel-1 Interferometric Wide Swath data, including 922 Sentinel-1 tiles covering the River Severn basin (UK) and the Lake Maggiore area (Italy). The results show that our exclusion map presents a good agreement $(\sim 63 \%)$ with reference data derived from different data sources and indicate that it may complement SAR-derived flood extent maps. Allowing to accurately identify potential misclassifications in flood extent mapping, our exclusion map provides valuable information for flood management and, in particular, flood forecasting and prediction.
\end{abstract}

\section{INTRODUCTION}

Flooding is a major hazard in both rural and urban areas worldwide, leading to significant human and economic losses. In order to reduce property damages and save people's lives, near real-time flood mapping is needed as it plays a central role in flood risk management in all dimensions, such as emergency response, flood forecasting, post-disaster reconstruction. In addition, flood maps derived from historical and up-to-date data can be employed for the (re-)calibration and validation of hydraulic models (Di Baldassarre et al., 2009). Moreover, such maps can be assimilated into flood forecasting systems in order to improve near real-time model-based predictions (Hostache et al., 2018).

Over the past decades, flood mapping using SAR data has gained considerable attention since the SAR systems can operate independently of sun illumination and weather conditions. Among a variety of flood mapping algorithms, change detection is widely used (Chini et al., 2017; Li et al., 2018; Schlaffer et al., 2015; Zhao et al., 2019) as the appearance of floodwater results most of the time in a drop of SAR backscattering. Generally, open calm water has low backscatter compared with surrounding land surfaces in a SAR intensity image. Change detection can, therefore, detect and map the presence of floodwater in a given SAR image by exploiting the changes with respect to a dryer reference image. This allows not only classifying water bodies but also distinguishing transient floodwater from permanent water bodies. However, the underlying assumption that floodwater leads to a decrease of the SAR intensity has some limits, especially in areas where the SAR signal does not reach the ground. For example, SAR cannot measure any floodingrelated changes in so-called shadow areas caused by prominent topography or obstacles on the ground (e.g. buildings). Moreover, the SAR signal cannot reach the ground in densely vegetated forests because of the low capability of SAR C-band wavelength to penetrate canopies (Tsyganskaya et al., 2018). It is noteworthy, in the latter case, L-band is more suitable given the higher capability of longer wavelengths to penetrate canopies. Additionally, there are some land cover classes where SAR can sense the surface but scattering variation caused by the presence of water is negligible when compared to the normal "unflooded" condition. Examples of such land cover classes are layover areas, dry sand, streets and building areas. In such cases, conventional change detection approaches fail in detecting floodwater.

However, it is surprising that only a handful of studies investigated the so-called exclusion areas. For instance, (Benoudjit and Guida, 2019) masked out shadow/layover areas from SAR images before generating flood maps with the help of a free Digital Elevation Model (DEM). When it comes to global scale flood mapping, high resolution DEMs are not always available and therefore, DEM-based shadow/layover maps may not be accurate enough in comparison with the SAR spatial resolution. Moreover, modelling shadow and layover caused by buildings and tall vegetation requires DEMs with metric spatial resolutions. It is of high importance to note that in built-up areas precise and timely information about the flood impact is required in order to save human lives. Change detection methods (Benoudjit and Guida, 2019; Zhao et al., 2019), which are designed for bare soil or scarcely vegetated scenarios, usually under-detect floodwater in urban areas since the backscattering decreasing assumption is no longer valid. Instead, the floodwater presence in urban areas often results in a backscattering increase (Mason et al., 2014). However, this phenomenon only exists up to a limited extent and, in this case, approaches exploiting multitemporal InSAR coherence may be more adequate for detecting urban floodwater (Chini et al., 2019). The case of dense forests where SAR signals cannot penetrate the canopy and reach the ground has been considered in (Bauer-Marschallinger et al., 2019). In their study, a sensitivity mask has been generated in order to identify regions with unreliable Sentinel-1-based surface

Corresponding author 
soil moisture (SSM). The mask includes densely forested areas as well as urban areas. However, since this sensitivity mask was designed for $500 \mathrm{~m}$ resolution SSM products, it may not be suitable for masking high to median-resolution SAR-based flood extent maps such as those derived from $20 \mathrm{~m}$ resolution Sentinel1 images. In order to deal with water-lookalike areas such as arid areas, (Martinis, 2018; Martinis et al., 2018) generated a Sand Exclusion Layer (SEL) using time-series of Sentinel-1 data for improving flood map accuracy.

In the above-mentioned studies, different masks for specific exclusion layers were generated for specific use cases. However, a comprehensive map/mask including all of the above-mentioned scenarios and tailored for SAR-based change detection approaches designed for flood mapping over bare soils and scarcely vegetated areas does not currently exist. With this aim, we propose an algorithm able to extract an "exclusion" map (EXmap), which is composed of all the pixels that cannot be classified as flooded or not using SAR-based change detection algorithms designed for bare soil and scarcely vegetated areas. The EX-map is extracted from time series of SAR data obtained from the same orbit. We argue that the availability of such an accompanying layer is important in any flood mapping exercise as it provides critical information for enabling a correct use of the data. For example, exclusion maps are required when EO-derived flood maps are assimilated into flood prediction models as any error in an assimilated flood map would have negative consequences. The proposed method is tested on stacks of Sentinel-1 intensity data at $20 \mathrm{~m}$ resolution acquired over different AOIs in the United Kingdom and Italy. In the considered test cases, the exclusion map is composed of the following classes: topographic shadow/layover, permanent water bodies, building shadow/double-bounce and densely vegetated forests. Due to the uniqueness of the proposed exclusion map, a similar and independent map/mask for cross-comparison is currently not available. In order to close this gap, an independent cross-comparison map is obtained by combining different products including 1) topographic shadow/layover map generated using a 25m-resolution DEM and the geometric acquisition parameters of the SAR data; 2) permanent water map; 3 ) imperviousness map; 4) tree cover density (TCD) map. The last three classes have a spatial resolution of 20 meters and are provided in the framework of the Copernicus programme.

The paper is organized as follows: the following section introduces two case studies and the associated datasets. The multi-temporal and spatial indices employed for deriving the exclusion map and the proposed algorithm are described in section 3. The results are presented, discussed and validated in section 4. Finally, a conclusion and an outlook on ongoing studies are provided in the final section.

\section{STUDY SITE AND DATASETS}

\subsection{Study sites}

Our first study site is located in the River Severn basin (UK) and the second one covers the Lake Maggiore area (Italy) as shown in Fig. 1. The two areas have been selected due to their different land cover classes and different topography, which are of high interest for the exclusion map identification.

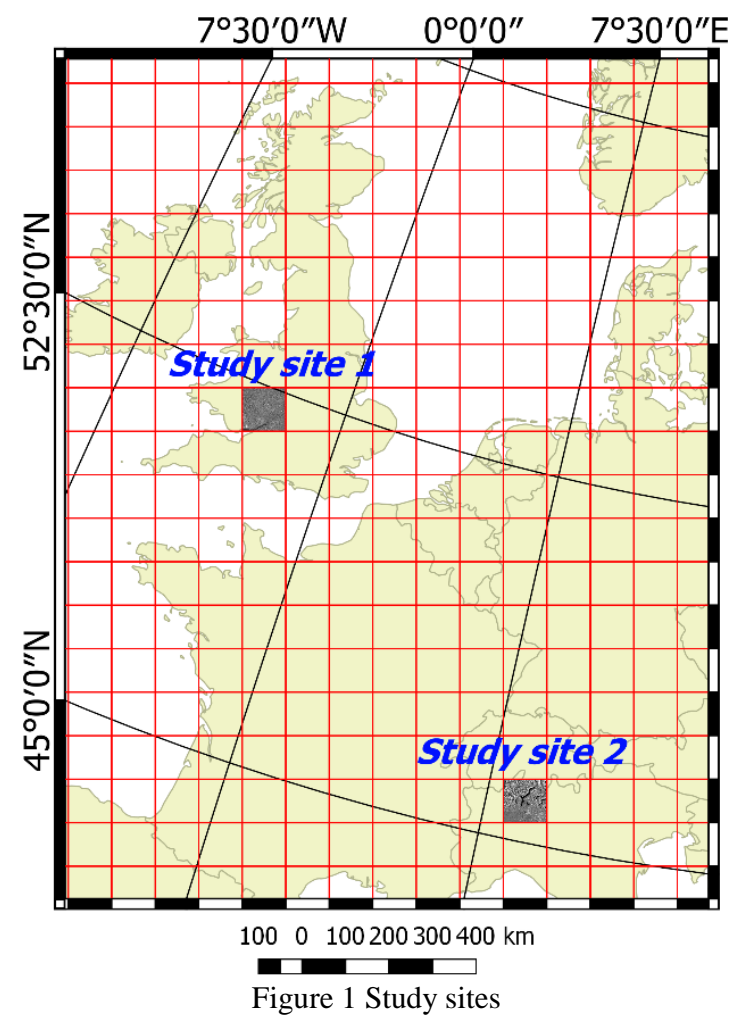

\subsection{SAR datasets}

The multi-temporal SAR datasets are composed of Sentinel-1 Interferometric Wide Swath (IW) data with VV polarization provided by TU Wien Data Cube (Ali et al., 2017). The latter is managed and processed on the Earth Observation Data Centre (EODC) for Water Resources Monitoring. The EODC uses the high-performance computing platform provided by the third generation of the Vienna Scientific Cluster (VSC-3), preparing easy access to EO data (Naeimi et al., 2016). In addition, EODC users can process EO data with their own algorithms and extract the results (Mathieu and Aubrecht, 2018). The Sentinel-1 data cube from TU Wien is derived by geocoding the SAR backscatter imagery using the python-based SAR Geophysical Retrieval Toolbox (SGRT) and the Sentinel-1 time-series from this data cube can be analysed directly in our study. The SAR datasets use the Equi7Grid basic framework (Bauer-Marschallinger et al., 2014) and all Sentinel-1 SAR data have been divided into $100 \mathrm{~km}$ $\times 100 \mathrm{~km}$ tiles as is shown in Fig. 1 .

\begin{tabular}{|c|c|c|c|c|}
\hline $\begin{array}{c}\text { Study } \\
\text { site }\end{array}$ & Equi7Grid & Track & Orbit & $\begin{array}{c}\text { Number of } \\
\text { images }\end{array}$ \\
\hline \multirow{2}{*}{1} & E040N023T1 & 30 & Ascending & 215 \\
& E040N023T1 & 154 & Descending & 282 \\
2 & E046N014T1 & 15 & Ascending & 167 \\
& E046N014T1 & 66 & Descending & 258 \\
\hline
\end{tabular}

Table 1 Information details of the employed Sentinel-1 IW datasets

In this paper, we use a total of 922 Sentinel-1 IW tiles with $20 \mathrm{~m}$ spatial resolution from four different tracks (Table 1). The acquisition time is between 2016 and 2018. 


\subsection{Reference datasets}

For the evaluation, different data sources have been combined for producing a map to compare with the unique exclusion map. The reference map is composed of the following classes:

(1) shadow and layover map from mountainous areas generated using $\quad$ a $25 \mathrm{~m} \quad$ resolution $\quad$ DEM (https://land.copernicus.eu/imagery-in-situ/eu-dem) and by considering the SAR geometry acquisition parameters for each particular Sentinel-1 orbit;

(2) 20m resolution imperviousness map representative of urban areas, provided by Copernicus and derived from high resolution optical satellite images (IRS-P6/Resourcesat-2 LISS-III, SPOT 5 and Landsat 8) (https://land.copernicus.eu/pan-european/highresolution-layers/imperviousness);

(3) $20 \mathrm{~m}$ resolution tree cover density (TCD) map representative of densely vegetated areas, derived from high resolution optical satellite data (https://land.copernicus.eu/pan-european/highresolution-layers/forests);

(4) $20 \mathrm{~m}$ resolution permanent water map derived from a multitude of SAR and optical EO data, provided in the Water \& Wetness 2015 Copernicus product. (https://land.copernicus.eu/pan-european/high-resolutionlayers/water-wetness).

\section{METHODOLOGY}

In this study, we define the exclusion map based on multitemporal stack of SAR images from the same orbit. We assume that all pixels presenting a stable backscatter over time shall be included in the mask. Such behaviour would prevent the detection of floodwater. Thus, our hypothesis is that some of the areas where floodwater cannot be detected using change detection approaches correspond to areas with markedly low SAR backscatter variations over time. Moreover, we assume that the targeted exclusion layer includes: (1) areas with extremely low backscatter over time (e.g. shadow areas, arid regions and permanent water bodies), (2) areas with extremely high backscatter over time (e.g. layover areas), (3) urban areas and (4) densely vegetated regions with moderate backscatter.

In this context, it is worth noting that each land cover class has its own speckle distribution and different spatial patterns. Consequently, the definition of a unique and general rule/parameter to extract the desired exclusion map and to characterize the temporal stability of the backscattering for different land cover classes is not straightforward. For this reason, we argue that the characteristics of different land cover classes need to be analysed separately.

In this study, time series Sentinel-1 data are analysed and five parameters are employed in our method: multi-temporal standard deviation (MSD), the multi-temporal median (MM), the multitemporal minima $(\mathrm{Mm})$, local Getis-Ord $G_{i}$ and data range. The latter two texture parameters are calculated from MM. Fig. 1 and Fig 2 show examples for different parameters derived from timeseries Sentinel-1 data covering Lake Maggiore areas in Italy and River Severn basin (UK) with corresponding optical images from Google Earth.
When it comes to areas with extremely low backscatter over time (e.g. shadow areas, arid regions and permanent water bodies), we propose to use a texture index, i.e. local Getis-Ord $G_{i}$ (Gamba et al., 2011; Getis and Ord, 1992) since such areas are considered to be homogeneous with extremely low backscatter over time. The local Getis-Ord $G_{i}$ is defined as:

$$
G_{i}=\frac{\sum_{j} w_{i j} x_{j}}{\sum_{j} x_{j}}, j \neq i
$$

where $\quad w_{i j}=$ the weight between $\mathrm{i}$ and $\mathrm{j}$ that represents their spatial relationship $x_{j}=$ the value of variable $\mathrm{x}$ at location $\mathrm{j}$

As is shown in equation (1), local Getis-Ord $G_{i}$ can be used to identify "outliers", i.e. values that are very different from the surrounding pixels. The high concentration of such values leads to high $G_{i}$ while a low concentration gives low $G_{i}$.

Meanwhile, the layover areas are supposed to be homogenous but with very high backscatter over time. Because of this characteristic, the local Getis-Ord $G_{i}$ can also be used for the extraction of layover in an analogous manner. As illustrated in Fig. 1d, permanent water bodies and shadow are associated with low $G_{i}$ values and are shown in black and dark blue while layover areas have high $G_{i}$ values and are shown in red. Hence, the identification of permanent water bodies, shadow and layover can be effectively performed by thresholding the local Getis-Ord $G_{i}$ values. In this preliminary study, thresholds have been selected by trial and error and visual inspection.

When considering urban areas, the double bounce effect between the buildings' facades and the ground occurs locally. Consequently, urban areas are very heterogeneous regions when compared to most other land cover classes in SAR images. To identify strongly heterogeneous areas, the texture parameter data range (Anys et al., 1998) based on MM is employed. This parameter is defined as the difference between the maximum value and minimum values within a sliding window. The strongly heterogeneous backscatter leads to high data range value while homogeneous areas result in low data range value. As is shown in Fig. 2e, the urban areas illustrated in red correspond to high data range values, which can be identified by thresholding.

For the densely vegetated regions with moderate backscatter variations, different indices, enabling the characterization of their temporal pixel backscatter stabilities or their relative higher or lower values with respect to pixels from other land cover classes, have been considered. In particular, three pixel-based multitemporal indexes (see Fig. 2a, b, c and Fig 3a, b, c) have been calculated, namely MSD, MM and Mm. Looking at the MSD map in Fig. 3a, MM map in Fig. 3b, Mm map in Fig. 3c and the optical image in Fig. 3d, they show that low MSD values, relatively high $\mathrm{MM}$ and relatively high $\mathrm{Mm}$ correspond to densely vegetated areas. Sparsely vegetated areas have higher MSD, relatively low MM and relatively low $\mathrm{Mm}$. Therefore, the densely vegetated areas can be distinguished from sparsely vegetated areas using thresholding on MSD, MM and Mm. It is important to notice that the identification of densely vegetated areas is carried out after removing areas with extremely low/high backscatter over time and urban areas. 

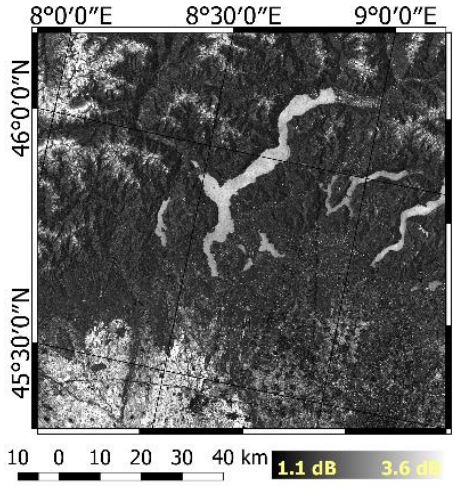

(a) Multitemporal Standard deviation

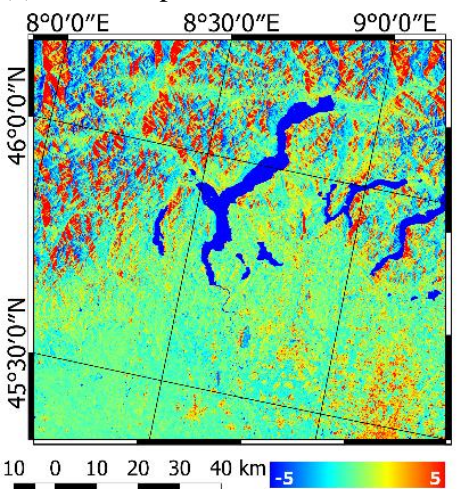

(d) Getis-Ord Gi
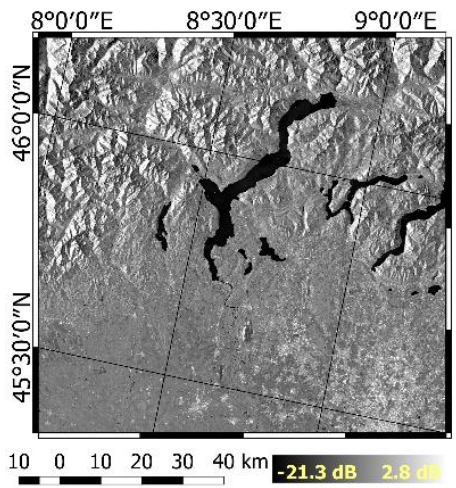

(b) Multitemporal Median

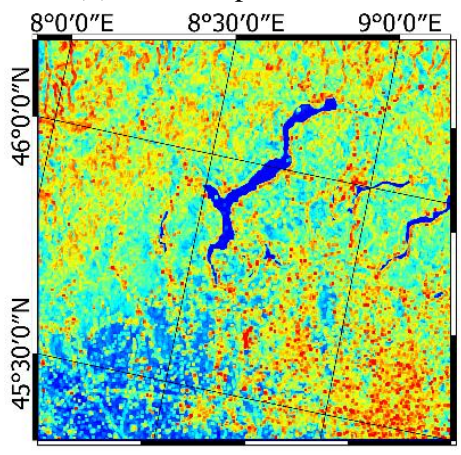

$10 \quad \begin{array}{llllll}0 & 10 & 20 & 30 & 40 & \mathrm{~km} \\ \mathbf{3} & \mathbf{d B} & 33 \mathrm{~dB}\end{array}$

(e) Data Range
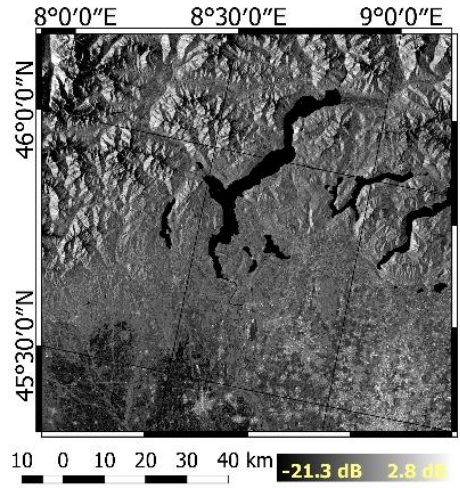

(c) Multitemporal Minimum

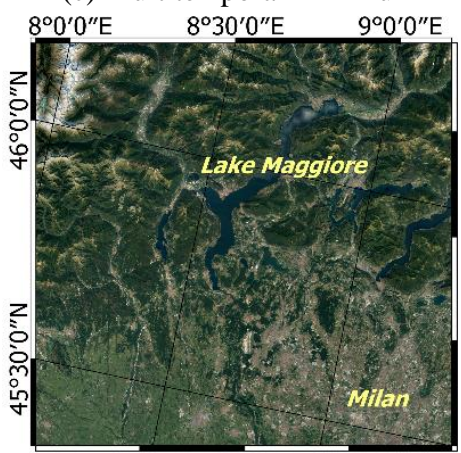

$\begin{array}{lllllll}10 & 0 & 10 & 20 & 30 & 40 & \mathrm{~km}\end{array}$

(f) Google Satellite Imagery

Figure 2 Example of multi-temporal parameters and indexes derived from time series Sentinel-1 image (E046N014T1, Track 15)

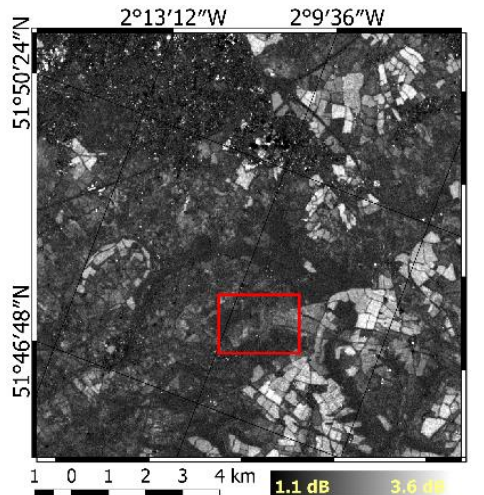

(a) Multitemporal Standard deviation

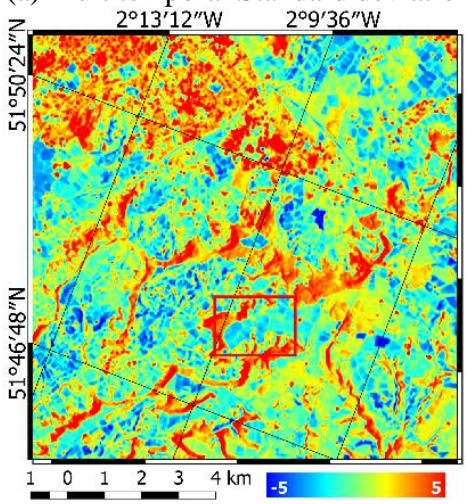

(d) Getis-Ord Gi

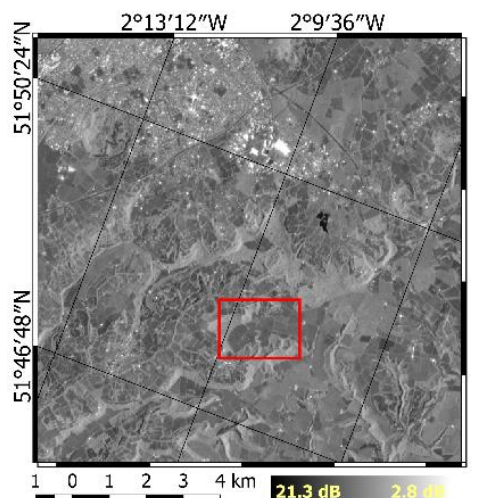

(b) Multitemporal Median

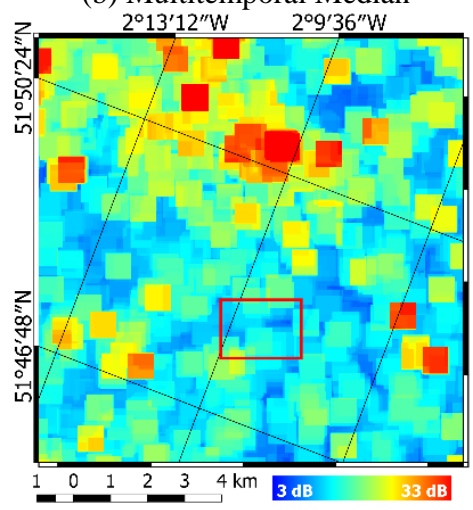

(e) Data Range

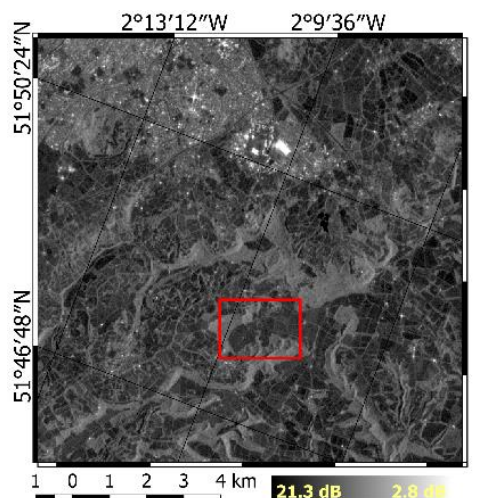

(c) Multitemporal Minimum

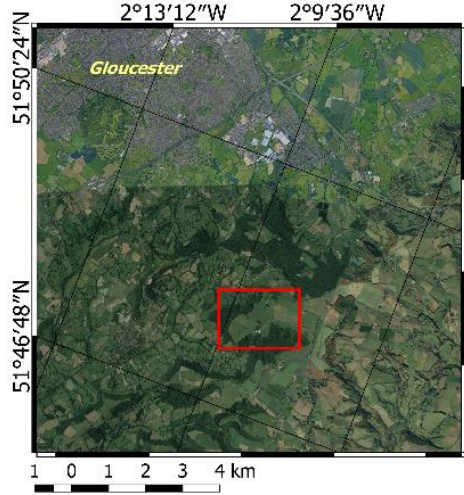

(f) Google Satellite Imagery

Figure 3 Examples of parameters derived from time series Sentinel-1 image (E040N023T1, track 30) for densely vegetated areas identification. Densely vegetated areas and sparsely vegetated areas are shown in the red box. 


\section{EXPERIMENTAL RESULTS}

Fig. 4 shows four exclusion maps (a-d) derived from four tracks and their corresponding reference maps (e-h) derived as explained in section 3.3. Based on visual inspection, we notice that our exclusion maps have a good agreement with the reference maps. Furthermore, we have performed a statistical comparison between the SAR derived and reference exclusion maps by computing the overall percent agreement. The overall agreement (Table 2) values ranging from $63 \%$ to $77 \%$ indicate that the SAR and reference exclusion maps present a relatively high percentage of similarities. The remaining disagreement can be explained in part by the diverse characteristics of the reference maps, using different data sources (e.g. SAR, optical data) of different spatial resolutions. Moreover, it is worth considering that classes composing the reference map are not representing exactly the same information content as the proposed exclusion map. Considering only urban areas, our approach identifies pixels associated with buildings double-bounce and associated shadow, while in the independent product used for comparison urban area class includes more classes such as parking lots, roads, small parks, etc. Furthermore, regarding densely vegetated areas, we can notice from Fig. 5 that the TCD class is absent in some areas, while a visual inspection of very high-resolution optical data
(Google Satellite Imagery) over the same areas indicates the presence of dense vegetation.

\begin{tabular}{|c|c|}
\hline & OA \\
\hline Track 30 & $76.7 \%$ \\
Track 154 & $77.9 \%$ \\
Track15 & $63.2 \%$ \\
Track 66 & $63.7 \%$ \\
\hline
\end{tabular}

Table 2 Sensitivity of exclusion maps derived from four study data tracks

By assessing the statistical comparison, we may also notice that the overall accuracy varies depending on the different test cases. As is shown in Table 2, the OA for track 30 and track 154 are $76.7 \%$ and $77.9 \%$ respectively while the OA for track 15 and track 66 are $63.2 \%$ and $63.7 \%$ respectively. This is due to the fact that the two study sites are composed of different land cover classes. In the study site in the UK, the vegetated areas contain sparsely vegetated areas such as grassland, pasture and orchards and densely vegetated areas such as dense forests. While in the second study site the Lake Maggiore area, most of the vegetated areas are dense forests and agriculture fields. We consider this as a preliminary analysis and evaluation of our exclusion map. Further tests performed for other AOIs are necessary in order to prove robustness of the method.

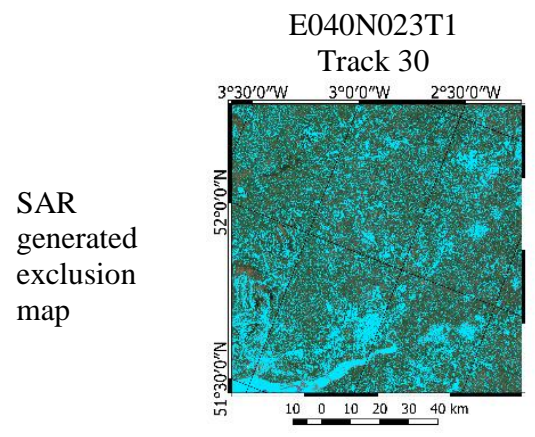

(a)

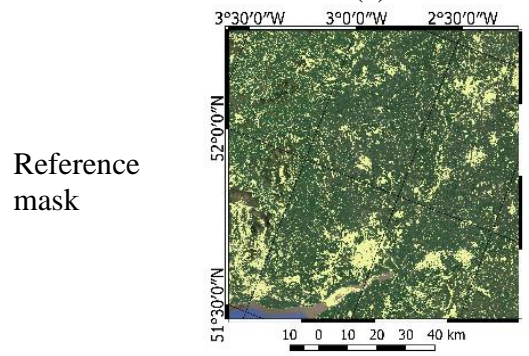

(e)

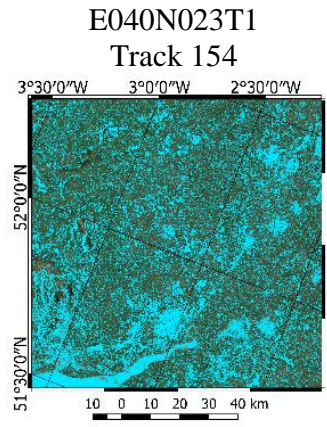

(b)

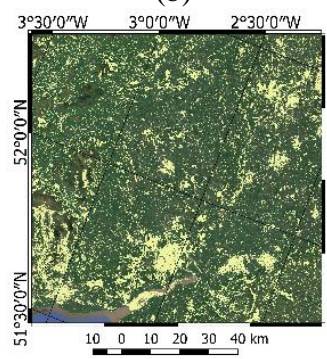

(f)
E046N014T1

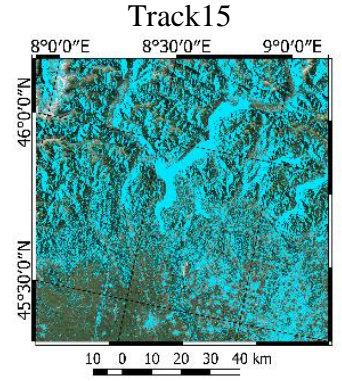

(c)

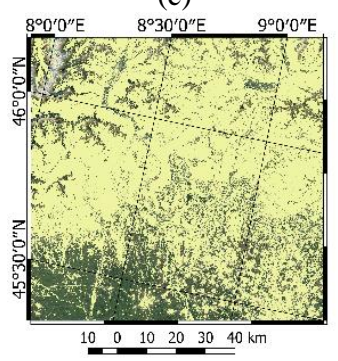

(g)
E046N014T1

Track 66

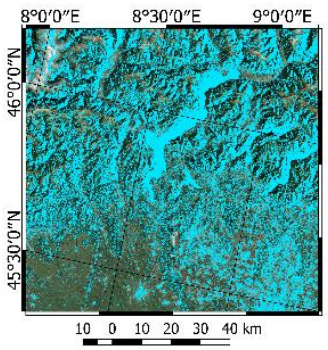

(d)

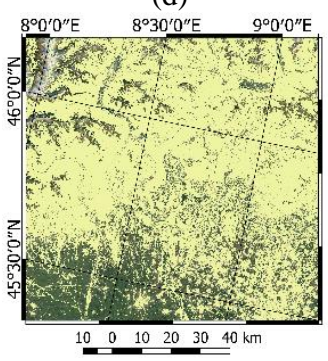

(h)

Figure 4 Generated exclusion maps from four tracks over two study sites

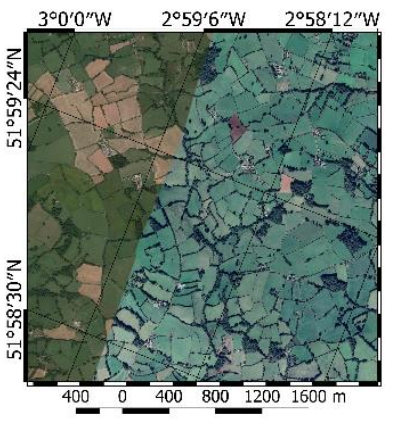

(a) Google Satellite Imagery

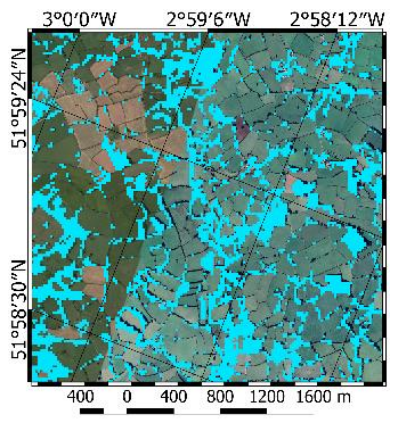

(b) $20 \mathrm{~m}$ resolution Exclusion Map

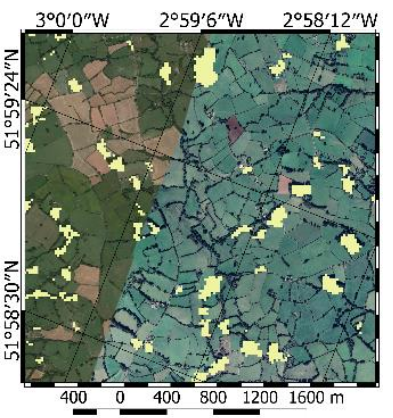

(c) $20 \mathrm{~m}$ resolution Reference $\square$ SAR generated exclusion map

Reference mask

Figure 5 Example of disagreement between the exclusion map derived from time series Sentinel-1 image (E040N023T1, track 30) and the reference map 


\section{CONCLUSION}

In this study, an exclusion map for flood extent maps has been generated by using $20 \mathrm{~m}$ resolution time-series Sentinel-1 intensity data over Severn River, UK and Maggiore Lake, Italy. The results show that our exclusion map has a good agreement with a reference map extracted from different data sources.

Improvements of the method are already foreseen for what it concerns the evaluation of the proposed method for additional different test cases. The automation of the method will also be improved by testing automatic and adaptive thresholding approaches. Alternative classification methods will be also tested. The impact of the SAR-based exclusion map on real flood scenarios will be evaluated, in terms of under detection of the maximum floodwater extent. Strategies to classify pixels from exclusion maps using other approaches and information will be envisaged. The benefit of this new type of information for what it concerns the calibration, validation and regular updating of hydraulic models using SAR-derived flood extent maps will be considered as well.

\section{ACKNOWLEDGEMENTS}

This work was supported by the Luxembourg National Research Fund (FNR) (reference: FNR PRIDE HYDRO-CSI 10623093).

\section{REFERENCES}

Ali, I., Naeimi, V., Cao, S., Elefante, S., Le, T., BauerMarschallinger, B., Wagner, W., 2017. Sentinel-1 data cube exploitation: Tools, products, services and quality control, in: 2017 Conference on Big Data from Space. https://doi.org/10.2760/383579

Anys, H., Bannari, A., He, D.-C., Morin, D., 1998. Cartographie des zones urbaines a l'aide des images aeroportees MEIS-II. Int. J. Remote Sens. 19, 883-894. https://doi.org/10.1080/014311698215775

Bauer-marschallinger, B., Freeman, V., Cao, S., Paulik, C., Schaufler, S., Stachl, T., Modanesi, S., Massari, C., Ciabatta, L., Brocca, L., Wagner, W., 2019. Towards Global Soil Moisture Monitoring with Sentinel-1 : Harnessing Assets and Overcoming Obstacles. IEEE Trans. Geosci. Remote Sens. 57, 520-539. https://doi.org/10.1109/TGRS.2018.2858004

Bauer-Marschallinger, B., Sabel, D., Wagner, W., 2014. Optimisation of global grids for high-resolution remote sensing data. Comput. Geosci. 72, 84-93. https://doi.org/10.1016/j.cageo.2014.07.005

Benoudjit, A., Guida, R., 2019. A Novel Fully Automated Mapping of the Flood Extent on SAR Images Using a Supervised

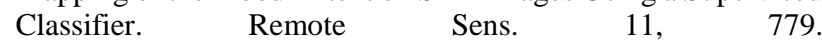
https://doi.org/10.3390/rs11070779

Chini, M., Hostache, R., Giustarini, L., Matgen, P., 2017. A Hierarchical Split-Based Approach for Parametric Thresholding of SAR Images: Flood Inundation as a Test Case. IEEE Trans. Geosci. Remote Sens. 55, 6975-6988. https://doi.org/10.1109/TGRS.2017.2737664

Chini, M., Pelich, R., Pulvirenti, L., Pierdicca, N., Hostache, R., Matgen, P., 2019. Sentinel-1 InSAR Coherence to Detect Floodwater in Urban Areas: Houston and Hurricane Harvey as A Test Case. Remote Sens. 11, 107. https://doi.org/10.3390/rs11020107

Di Baldassarre, G., Schumann, G., Bates, P.D., 2009. A technique for the calibration of hydraulic models using uncertain satellite observations of flood extent. J. Hydrol. 367, 276-282. https://doi.org/10.1016/j.jhydrol.2009.01.020

Gamba, P., Aldrighi, M., Stasolla, M., 2011. Robust Extraction of Urban Area Extents in HR and VHR SAR Images. IEEE J. Sel. Top. Appl. Earth Obs. Remote Sens. 4, 27-34. https://doi.org/10.1109/JSTARS.2010.2052023

Getis, A., Ord, J.K., 1992. The Analysis of Spatial Association by Use of Distance Statistics. Geogr. Anal. 24, 189-206. https://doi.org/10.1111/j.1538-4632.1992.tb00261.x

Hostache, R., Chini, M., Giustarini, L., Neal, J., Kavetski, D., Wood, M., Corato, G., Pelich, R., Matgen, P., 2018. Near-RealTime Assimilation of SAR-Derived Flood Maps for Improving Flood Forecasts. Water Resour. Res. 54, 5516-5535. https://doi.org/10.1029/2017WR022205

Li, Y., Martinis, S., Plank, S., Ludwig, R., 2018. An automatic change detection approach for rapid flood mapping in Sentinel-1 SAR data. Int. J. Appl. Earth Obs. Geoinf. 73, 123-135. https://doi.org/10.1016/j.jag.2018.05.023

Martinis, S., 2018. A Sentinel-1 Times Series-Based Exclusion Layer for Improved Flood Mapping in Arid Areas, in: IGARSS 2018 - 2018 IEEE International Geoscience and Remote Sensing Symposium. IEEE, pp. 6584-6587. https://doi.org/10.1109/IGARSS.2018.8518604

Martinis, S., Plank, S., Ćwik, K., 2018. The Use of Sentinel-1 Time-Series Data to Improve Flood Monitoring in Arid Areas. Remote Sens. 10, 583. https://doi.org/10.3390/rs10040583

Mathieu, P., Aubrecht, C., 2018. Earth Observation Open Science and Innovation, Earth Observation Open Science and Innovation. Springer International Publishing, Cham. https://doi.org/10.1007/978-3-319-65633-5

Naeimi, V., Wagner, W., Elefante, S., Dostalova, A., Cao, S., Bauer-Marschallinger, B., 2016. Geophysical Parameters Retrieval From Sentinel-1 Sar Data: A Case Study For High Performance Computing At EODC, in: 24th High Performance Computing Symposium. Society for Modeling and Simulation International (SCS), pp. 68-75. https://doi.org/10.22360/SpringSim.2016.HPC.026

Schlaffer, S., Matgen, P., Hollaus, M., Wagner, W., 2015. Flood detection from multi-temporal SAR data using harmonic analysis and change detection. Int. J. Appl. Earth Obs. Geoinf. 38, 15-24. https://doi.org/10.1016/j.jag.2014.12.001

Tsyganskaya, V., Martinis, S., Marzahn, P., Ludwig, R., 2018. SAR-based detection of flooded vegetation - a review of characteristics and approaches. Int. J. Remote Sens. 39, 22552293. https://doi.org/10.1080/01431161.2017.1420938

Zhao, J., Chini, M., Matgen, P., Hostache, R., Pelich, R., Wagner, W., 2019. An Automatic SAR-Based Change Detection Method for Generating Large-Scale Flood Data Records: The UK as a Test Case, in: IGARSS 2019 - 2019 IEEE International Geoscience and Remote Sensing Symposium. IEEE, pp. 61386141. https://doi.org/10.1109/IGARSS.2019.8900534 